\title{
Magnon-magnon interaction and magnon relaxation time in ferromagnetic
}

\section{$\mathrm{Cr}_{2} \mathrm{Ge}_{2} \mathrm{Te}_{6}$ monolayer}

Ke Wang ${ }^{1,2}$, Xiansong $\mathrm{Xu}^{3}$, Yuan $\mathrm{Cheng}^{2}$, Min Zhang ${ }^{1}$, Jian-Sheng Wang ${ }^{4}$, Hai Wang ${ }^{1 *}$, and Gang Zhang $^{2 *}$

1) Xidian University, No. 2 Taibai Road, Xi’an, Shaanxi Province 710071, China

2) Institute of High Performance Computing, A*STAR, 138632, Singapore

3) Science, Mathematics and Technology Cluster, Singapore University of Technology and

Design, 487372, Singapore

4) Department of Physics, National University of Singapore, 117551, Singapore

Email: wanghai@mail.xidian.edu.cn; zhangg@,ihpc.a-star.edu.sg

\begin{abstract}
Despite the intense amount of attention and huge potential of two-dimensional (2D) magnets for applications in novel magnetic, magneto-optical, magneto-thermal and magneto-electronic devices, there has yet to be a robust strategy developed to systematically understand magnon-magnon (MMI) interactions at finite temperature. In this paper, we present a first-principles theoretical method to introduce the finite temperature magnon-magnon interaction into Heisenberg Hamiltonian through a nonlinear correction energy. The Wick's theorem is used to decouple the four-magnon operators to twomagnon order. We demonstrate the capabilities of this method by studying the strength of MMI in $\mathrm{Cr}_{2} \mathrm{Ge}_{2} \mathrm{Te}_{6}(\mathrm{CGT})$ monolayer. The spin wave spectrum at finite temperature and the time-dependent spin autocorrelation function are explored. It is found that the magnon relaxation time due to magnon-magnon scattering increases with temperature because of the reduction in magnon energy, while decreases with wavevector and external magnetic field. Our results provide a new insight to understand the magnon damping and energy dissipation in two-dimensional ferromagnetic materials.
\end{abstract}

KEYWORDS: Two-dimensional magnets; Ferromagnetism; Magnon-magnon interaction; Spin-wave spectrum 


\section{Introduction}

The two-dimensional (2D) materials exfoliated from various bulk van der Waals sheets (vdW) attract substantial attention in materials science, condensed matter physics, and electronic engineering, because of their fascinating properties[1-4]. Although all major electronic classes (metals, insulators, and semiconductors) have been observed in 2D materials[5-7], 2D magnet was missing until the discovery of intrinsic ferromagnetism in $\mathrm{Cr}$ trihalide and chalcogenides in 2017[8,9]. Subsequently, numerous 2D magnets with long-range order have been discovered and investigated, such as $\mathrm{Fe}_{5-\mathrm{x}} \mathrm{GeTe}_{2}[10,11], \mathrm{XPS}_{3}$ $(\mathrm{X}=\mathrm{Cr}, \mathrm{Mn}, \mathrm{Fe})[12-16], \mathrm{CrPS}_{4}, \mathrm{Cr}_{2} \mathrm{~S}_{3}[17,18], \mathrm{CoGa}_{2} \mathrm{X}_{4}(\mathrm{X}=\mathrm{S}, \mathrm{Se}, \mathrm{Te})[19], \mathrm{XH}_{2}(\mathrm{X}=\mathrm{Sc}, \mathrm{Ti}, \mathrm{V}, \mathrm{Cr}, \mathrm{Fe}$ $\mathrm{Co}, \mathrm{Ni})$ [20], $\mathrm{CrOX}(\mathrm{X}=\mathrm{Cl}, \mathrm{Br})[21,22]$, non-vdW transition-metal oxides[23] and some 2D transition metal carbides and nitrides (MXenes)[24,25].

For 2D magnets, magnetic anisotropy including the easy axis, the Kitaev interaction and the single ion anisotropy is of vital importance because sizeable MAE is beneficial to the long-range ferromagnetic order at finite temperature[26], so numerous researchers have been devoted to related study[27-31]. Based on the magnetic anisotropy, the Curie/ Neel temperature is predicted through the mean field theory. Meantime, several studies have been implemented to enhance the Curie/ Neel temperature for the application of 2D magnets in magneto-optical, magneto-thermal and magneto-electronic devices by functionalization[3234], external field[8,35] and strain[36,37]. Below the Curie/Neel temperature, the time-resolved magnetooptical Kerr effect (TR-MOKE) and the time-resolved Faraday rotation (TRFR) are often employed to investigate the magnetic-optical coupling[9,38,39], demonstrating the rich magnetic behaviors in 2D magnets. The Hall effect (including spin Hall effect, anomalous Hall effect and thermal Hall effect) in 2D magnets also attracts substantial attention, which not only promotes the development of novel Hall devices but also provides a new route to detect the existence of magnetism[40-44]. Among these Hall effects, the thermal Hall effect is related to the coupling between spin wave and lattice vibration (magnon-phonon 
coupling)[45], and the magnon-phonon coupling is usually studied by Raman spectra[46,47] phonon spectrum[48,49] and spin-wave spectrum[50,51]. In spintronics, the manipulation of magnetic order also is a hot topic, and there are numerous manipulation strategies, such as stack[52,53], electrostatic doping[54,55], and pressure[56,57]. So far, despite intense study in this area, there is a lack of a robust theoretical method for studying the magnon-magnon interaction (MMI) at finite temperature, which is crucial for the coherent spintronics.

In this paper, we introduce the nonlinear MMI into Heisenberg Hamiltonian to describe the spinwave spectrum of CGT monolayer at finite temperature. The influence of nonlinear MMI on magnon dispersion can be regarded as a nonlinear correction energy caused by the variety in magnon population. To observe the MMI, the time-dependence spin autocorrelation is employed, and the relaxation time $\tau_{\mathrm{MM}}$ is obtained by fitting the spin autocorrelation with exponential function. We theoretically find the relaxation time $\tau_{\mathrm{MM}}$ (contributed by MMI) increase with temperature but decreases remarkably with the external magnetic field. Our results shed a light on the understanding of dissipation of magnon energy at finite temperature.

\section{Theoretical models and computational details}

Here, the ferromagnetic system is described by the Heisenberg Hamiltonian including the nearestneighbor $(\mathrm{N})$ exchange and Zeeman energy:

$$
H=-\frac{1}{2} \sum_{\substack{l, f \in \mathrm{N} \\ l \neq f}} J \boldsymbol{S}_{l} \cdot \boldsymbol{S}_{f}-\sum_{l} g \mu_{B} \boldsymbol{B} \cdot \boldsymbol{S}_{l} .
$$

For a given magnetic lattice, $S=\left(S^{x}, S^{y}, S^{z}\right)$ is the spin vector whose amplitude is $S_{0} . J$ is the exchange constant of the nearest-neighbor interaction, $g$ is Landé factor, $\mu_{B}$ is the Bohr magneton, and $\boldsymbol{B}$ represents an external magnetic field along the $c$-axis. Without loss of generality, the equilibrium magnetization is

assumed to parallel to the external field. In addition, transverse components $S^{ \pm}=S^{x} \pm i S^{y}$ are defined to 
eliminate the dependence between $S^{x}$ and $S^{y}$. According to Holstein-Primakoff (HP) approximation[58,59], $S^{ \pm}$and $S^{\mathrm{Z}}$ for a given site can be written as

$$
S^{+}=\left(\sqrt{2 S_{0}-a^{+} a}\right) a, S^{-}=a^{+}\left(\sqrt{2 S_{0}-a^{+} a}\right), S^{z}=\left(S_{0}-a^{+} a\right),
$$

where $a^{+}$and $a$ are the creation and annihilation operators of magnon for the given site, respectively. The operators of magnon described in coordinate space are transferred into reciprocal space by the Fourier transform:

$$
a_{k}=N^{-\frac{1}{2}} \sum_{r} e^{-i k \cdot r} a, a_{k}^{+}=N^{-\frac{1}{2}} \sum_{r} e^{i k \cdot r} a^{+}
$$

where $\boldsymbol{k}$ is the wave vector, $\boldsymbol{r}$ is the position vector of the lattice point, and $N$ is the number of unit cells in the supercell. The expectation value for local spin $\left\langle a_{k}^{+} a_{k}\right\rangle$ can be calculated by the Planck distribution function:

$$
\left\langle a_{k}^{+} a_{k}\right\rangle=\frac{1}{\left(e^{\hbar \omega_{k} / k_{B} T}-1\right)},
$$

with Boltzmann constant $k_{B}$ and the reduced Planck constant $\hbar$. At low temperature (close to zero), $\hbar \omega$ $/ k_{B} T$ tends to be infinite, resulting in an ignorable $\left\langle a_{k}^{+} a_{k}\right\rangle$. With temperature increases, $\left\langle a_{k}^{+} a_{k}\right\rangle$ increases significantly, thus cannot be ignored.

\subsection{Zero temperature model.}

For the completeness, firstly we introduce the spin spectrum model at zero temperature, at which the population of excited magnon is low, therefore the expectation value for local spin can be negligible with respect to $2 S_{0}[60,61]$. The transverse components of spin vector for a given site can be rewritten as:

$$
S^{+}=\left(\sqrt{2 S_{0}-a^{+} a}\right) a \approx\left(\sqrt{2 S_{0}}\right) a, S^{-}=\left(\sqrt{2 S_{0}-a^{+} a}\right) a^{+} \approx a^{+}\left(\sqrt{2 S_{0}}\right) .
$$


The Hamiltonian can be given as:

$$
H=H_{0}+H_{B}=-\frac{1}{2} J \sum_{\substack{l, f \in \mathrm{N} \\ l \neq f}}\left[\left(S_{0}-a_{l}^{+} a_{l}\right)\left(S_{0}-a_{f}^{+} a_{f}\right)+S_{0}\left(a_{l} a_{f}^{+}+a_{l}^{+} a_{f}\right)\right]-\sum_{l} g \mu_{B} B \cdot\left(S_{0}-a_{l}^{+} a_{l}\right),
$$

Here $H_{0}$ and $H_{B}$ are:

$$
\begin{gathered}
H_{0}=-\frac{1}{2} J \sum_{\substack{l, f \in \mathrm{N} \\
l \neq f}}\left[\left(S_{0}-a_{l}^{+} a_{l}\right)\left(S_{0}-a_{f}^{+} a_{f}\right)+S_{0}\left(a_{l} a_{f}^{+}+a_{l}^{+} a_{f}\right)\right], \\
H_{B}=-\sum_{l} g \mu_{B} B \cdot\left(S_{0}-a_{l}^{+} a_{l}\right) .
\end{gathered}
$$

Following Eq. (3), Eq. (6) can be transferred into the reciprocal space. Then, the Hamiltonian in the reciprocal space can be written as:

$$
H=H_{0}+H_{B}=-\frac{1}{2} J \sum_{\substack{l, f \in N \\ l \neq f}}\left(S_{0}^{2}-S_{0} N^{-1} \sum_{k}\left(a_{l, k}^{+} a_{l, k}+a_{f, k}^{+} a_{f, k}-a_{f, k}^{+} a_{l, k} \gamma_{k}-a_{l, k}^{+} a_{f, k} \gamma_{k}\right)\right)-\sum_{l} g \mu_{B} B \cdot\left(S_{0}-N^{-1} \sum_{k} a_{l, k}^{+} a_{l, k}\right)
$$

with $\gamma_{k}=\frac{1}{Z} \sum_{l, f \in \mathrm{N}} e^{i k \cdot\left(\boldsymbol{r}_{f}-\boldsymbol{r}_{l}\right)} . \mathrm{Z}$ is the coordination number for the nearest interaction.

In each unit cell of monolayer CGT, it includes two magnetic $\mathrm{Cr}^{3+}$ ions, then the Hamiltonian is:

$$
H=H_{0}+H_{B}=E_{0}+\sum_{k} \hbar \omega_{k}^{ \pm} a_{k}^{+} a_{k}=E_{0}+\sum_{k}\left(J Z S_{0}\left(1 \pm \gamma_{k}\right)+2 g \mu_{B} B\right) a_{k}^{+} a_{k}
$$

where $E_{0}$ is the energy of ground state:

$$
E_{0}=-2 N J Z S_{0}-2 N g \mu_{B} B S_{0} .
$$

In Eq. (10), the optical $\left(\hbar \omega^{+}\right)$and acoustic $\left(\hbar \omega^{-}\right)$branches of spin waves are gained when the plus and minus signs are taken, respectively.

\subsection{Magnon-magnon interaction at finite temperature.}

From Eq. (4), it is obvious that the expectation value for local spin increases with temperature because the excitation of substantial magnons, so that at finite temperature the transverse components of spin vector for a given site should be written as follows[62]: 


$$
S^{+}=\left(\sqrt{2 S_{0}-a^{+} a}\right) a \approx \sqrt{2 S_{0}}\left(1-\frac{a^{+} a}{4 S_{0}}\right) a, S^{-}=a^{+}\left(\sqrt{2 S_{0}-a^{+} a}\right) \approx a^{+} \sqrt{2 S_{0}}\left(1-\frac{a^{+} a}{4 S_{0}}\right)
$$

Then, the higher order terms induced by the nonlinear MMI are included in Heisenberg Hamiltonian:

$$
H(T) \approx H_{0}+H_{B}-\frac{1}{2} J \sum_{\substack{l, f \in N \\ l \neq f}}\left[a_{l}^{+} a_{l} a_{f}^{+} a_{f}+\frac{a_{l}^{+} a_{l} a_{l} a_{f}^{+}}{4}+\frac{a_{f}^{+} a_{f} a_{l} a_{f}^{+}}{4}+\frac{a_{l}^{+} a_{f} a_{l}^{+} a_{l}}{4}+\frac{a_{l}^{+} a_{f} a_{f}^{+} a_{f}}{4}\right] .
$$

Next, the normal coordinate is introduced into Hamiltonian by Eq. (3), and the Fourier transform for the four-order terms are shown as following:

$$
\begin{aligned}
& a_{l}^{+} a_{l} a_{f}^{+} a_{f}=N^{-2} \sum_{k-q} e^{-i(k-q) \boldsymbol{r}_{l}} a_{k-q}^{+} \sum_{k} e^{i k \boldsymbol{r}_{f}} a_{k} \sum_{k^{\prime}+q} e^{-i\left(k^{\prime}+q\right) \boldsymbol{r}_{f}} a_{k^{\prime}+q}^{+} \sum_{k^{\prime}} e^{i k^{\prime} \boldsymbol{r}_{l}} a_{k^{\prime}}=N^{-2} \sum_{k, k^{\prime}, q} \gamma_{k-k^{\prime}-q} a_{k-q}^{+} a_{k^{\prime}+q}^{+} a_{k^{\prime}} a_{k}, \\
& a_{l}^{+} a_{l} a_{l} a_{f}^{+}=N^{-2} \sum_{k-q} e^{-i(k-q) \boldsymbol{r}_{f}} a_{k-q}^{+} \sum_{k} e^{i k \boldsymbol{r}_{l}} a_{k} \sum_{k^{\prime}+q} e^{-i\left(k^{\prime}+q\right) \boldsymbol{r}_{l}} a_{k^{\prime}+q}^{+} \sum_{k^{\prime}} e^{i k^{\prime} \boldsymbol{r}_{l}} a_{k^{\prime}}=N^{-2} \sum_{k, k^{\prime}, q} \gamma_{k-q} a_{k-q}^{+} a_{k^{\prime}+q}^{+} a_{k^{\prime}} a_{k}, \\
& a_{f}^{+} a_{f} a_{l} a_{f}^{+}=N^{-2} \sum_{k-q} e^{-i(k-q) \boldsymbol{r}_{f}} a_{k-q}^{+} \sum_{k} e^{i k r_{f}} a_{k} \sum_{k^{\prime}+q} e^{-i\left(k^{\prime}+q\right) \boldsymbol{r}_{f}} a_{k^{\prime}+q}^{+} \sum_{k^{\prime}} e^{i k^{\prime} \boldsymbol{r}_{l}} a_{k^{\prime}}=N^{-2} \sum_{k, k^{\prime}, q} \gamma_{k^{\prime}} a_{k-q}^{+} a_{k^{\prime}+q}^{+} a_{k^{\prime}} a_{k}, \\
& a_{l}^{+} a_{f} a_{l}^{+} a_{l}=N^{-2} \sum_{k-q} e^{-i(k-q) \boldsymbol{r}_{l}} a_{k-q}^{+} \sum_{k} e^{i k \boldsymbol{r}_{l}} a_{k} \sum_{k^{\prime}+q} e^{-i\left(k^{\prime}+q\right) \boldsymbol{r}_{l}} a_{k^{\prime}+q}^{+} \sum_{k^{\prime}} e^{i k^{\prime} \boldsymbol{r}_{f}} a_{k^{\prime}}=N^{-2} \sum_{k, k^{\prime}, q} \gamma_{k^{\prime}} a_{k-q}^{+} a_{k^{\prime}+q}^{+} a_{k^{\prime}} a_{k}, \\
& a_{l}^{+} a_{f} a_{l}^{+} a_{l}=N^{-2} \sum_{k-q} e^{-i(k-q) \boldsymbol{r}_{l}} a_{k-q}^{+} \sum_{k} e^{i k \boldsymbol{r}_{l}} a_{k} \sum_{k^{\prime}+q} e^{-i\left(k^{\prime}+q\right) \boldsymbol{r}_{l}} a_{k^{\prime}+q}^{+} \sum_{k^{\prime}} e^{i k^{\prime} \boldsymbol{r}_{f}} a_{k^{\prime}}=N^{-2} \sum_{k, k^{\prime}, q} \gamma_{k^{\prime}} a_{k-q}^{+} a_{k^{\prime}+q}^{+} a_{k^{\prime}} a_{k}, \\
& a_{l}^{+} a_{f} a_{f}^{+} a_{f}=N^{-2} \sum_{k-q} e^{-i(k-q) \boldsymbol{r}_{l}} a_{k-q}^{+} \sum_{k} e^{i k \boldsymbol{r}_{f}} a_{k} \sum_{k^{\prime}+q} e^{-i\left(k^{\prime}+q\right) \boldsymbol{r}_{f}} a_{k^{\prime}+q}^{+} \sum_{k^{\prime}} e^{i k^{\prime} \boldsymbol{r}_{f}} a_{k^{\prime}}=N^{-2} \sum_{k, k^{\prime}, q} \gamma_{k-q} a_{k-q}^{+} a_{k^{\prime}+q}^{+} a_{k^{\prime}} a_{k} .
\end{aligned}
$$

Then, the Hamiltonian at finite temperature in reciprocal space can be represented by

$$
H(T)=H_{0}+H_{B}-J Z N^{-1} \sum_{k, k^{\prime}, q} a_{k-q}^{+} a_{k^{\prime}+q}^{+} a_{k^{\prime}} a_{k}\left(\gamma_{k-k^{\prime}-q}-\frac{\gamma_{k-q}}{2}-\frac{\gamma_{k^{\prime}}}{2}\right)
$$

There is no analytical solution for the four magnon operators, so it is necessary to decompose the products of four magnon operators. Wick's theorem, an algebraic strategy, states that the product of operators is equal to the sum of all possible pairs of operators, and allows one to handle the reduction 
problem quite easily. Here we adopt the Wick's theorem to decouple the four-magnon operators to twomagnon order[63]:

$$
a_{k-q}^{+} a_{k^{\prime}+q}^{+} a_{k^{\prime}} a_{k}=\left(a_{k-q}^{+} a_{k^{\prime}+q}^{+}\right)\left(a_{k^{\prime}} a_{k}\right)+\left(a_{k-q}^{+} a_{k^{\prime}}\right)\left(a_{k^{\prime}+q}^{+} a_{k}\right)+\left(a_{k-q}^{+} a_{k}\right)\left(a_{k^{\prime}+q}^{+} a_{k^{\prime}}\right) .
$$

Meantime, the low order Taylor approximation is used to simplify the Hamiltonian:

$$
\left(a_{k-q}^{+} a_{k^{\prime}}\right)\left(a_{k^{\prime}+q}^{+} a_{k}\right)=\left[\left\langle a_{k-q}^{+} a_{k^{\prime}}\right\rangle+\left(a_{k-q}^{+} a_{k^{\prime}}-\left\langle a_{k-q}^{+} a_{k^{\prime}}\right\rangle\right)\right] \cdot\left[\left\langle a_{k^{\prime}+q}^{+} a_{k}\right\rangle+\left(a_{k^{\prime}+q}^{+} a_{k}-\left\langle a_{k^{\prime}+q}^{+} a_{k}\right\rangle\right)\right] .
$$

Finally, the Hamiltonian at finite temperature can be written as:

$$
H(T)=H_{0}+H_{B}+E_{1}+J Z N^{-1} \sum_{k, k^{\prime}, q}\left(\left\langle a_{k-q}^{+} a_{k}\right\rangle a_{k^{\prime}+q}^{+} a_{k^{\prime}}+\left\langle a_{k^{\prime}+q}^{+} a_{k^{\prime}}\right\rangle a_{k-q}^{+} a_{k}+\left\langle a_{k-q}^{+} a_{k^{\prime}}\right\rangle a_{k^{\prime}+q}^{+} a_{k}+\left\langle a_{k^{\prime}+q}^{+} a_{k}\right\rangle a_{k-q}^{+} a_{k^{\prime}}\right)\left(\frac{\gamma_{k-q}}{2}+\frac{\gamma_{k^{\prime}}}{2}-2 \gamma_{k-k^{\prime}-q}\right)
$$

with $\sum_{k^{\prime}} \gamma_{k-k^{\prime}}=\gamma_{k} \sum_{k^{\prime}} \gamma_{k} \cdot E_{1}$ is the static energy difference from the ground state. Consequently, the magnon dispersion at finite temperature can be represented as:

$$
\hbar \omega_{k}^{ \pm}(T)=J Z S_{0}\left(1 \pm \gamma_{k}\right)\left(1-\frac{1}{N S_{0}} \sum_{k^{\prime}}\left(1-\gamma_{k^{\prime}}\right)\left\langle a_{k^{+}}^{+} a_{k^{\prime}}\right\rangle\right)+2 g \mu_{B} B
$$

Therefore, the effect of nonlinear MMI induced by temperature on spin-wave spectrum can be regarded as a correction term. This modification of the spin-wave spectrum can be carried out by a selfconsistent procedure.

\subsection{Computational details}

In this paper, we used the experimental lattice constants ( $a=b=6.83 \AA$ ) [64], and the geometrical structure is relaxed by the Vienna ab initio simulation package (VASP) [65] using 500 eV cutoff energy and generalized gradient approximation (GGA) with $U$ of 1 eV $[49,66]$. A vacuum space of $16 \AA$ is set along the out-of-plane direction, because it was predicted that CGT monolayer has an out-of-plane magnetic easy axis[49]. The convergence for the energy difference of self-consistent iterations and the Hellmann-Feynman force were $10^{-8} \mathrm{eV}$ and $0.001 \mathrm{eV} / \AA$, respectively. A $k$-point mesh of $5 \times 5 \times 1$ was used 
for structural relaxation, while a $9 \times 9 \times 1$ mesh was used for self-consistent calculation to obtain the energy of CGT unit cell under different magnetic configurations.

\section{Results and discussion}

\section{1 Spin-wave spectrum at finite temperature.}

(a)

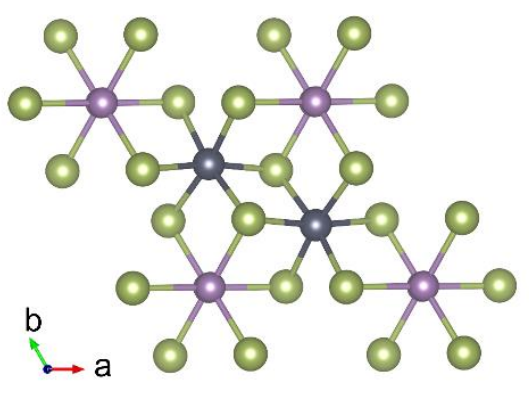

(b)

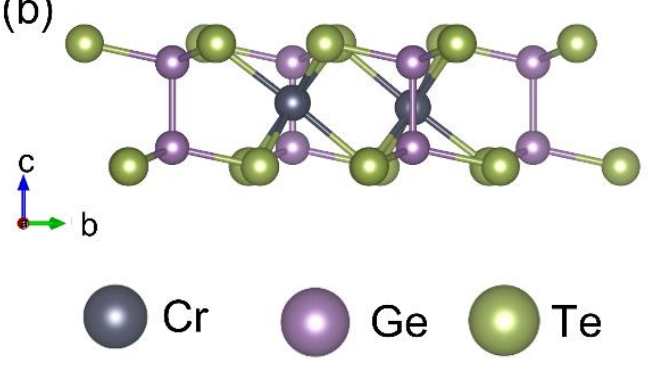

(c)

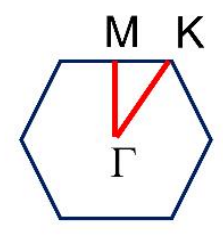

Figure 1. Top (a) and side (b) view of $\mathrm{Cr}_{2} \mathrm{Ge}_{2} \mathrm{Te}_{6}$ (CGT) monolayer. The red parallelogram in (a) marks out the unit cell. The $\mathrm{Cr}$, Ge and Te atoms are denoted in gray, purple and dark green colors, respectively. (c) The irreducible Brillouin zone of CGT monolayer.

The geometric structure and the path in Brillouin zone of CGT monolayer are shown in Figure 1. It was reported that Te atoms play a fundamental role in stabilizing the ferromagnetism of monolayer CGT through the super-exchange interactions [66-68], although they are nonmagnetic. In this work, only the nearest-neighboring interaction was taken into account, because the next-nearest-neighboring is one order of magnitude weaker[38,49]. The ferromagnetic ground state of CGT monolayer is confirmed, as the total energy of ferromagnetism (FM) phase is $-48.407 \mathrm{eV}$, and that of antiferromagnetic (AFM) phase is $-48.345 \mathrm{eV}$. The exchange constant can be calculated by:

$$
J=\left(E_{\mathrm{AFM}}-E_{\mathrm{FM}}\right) /\left(2 Z S_{0}^{2}\right) .
$$

In CGT monolayer, the coordinate number of nearest-neighboring interaction is 3. At zero temperature, 
the exchange constant is $4.557 \mathrm{meV}$. In the following section, we would use the exchange constant $J$ at zero temperature to calculate the magnon dispersion and investigate the MMI, following the previous studies[69,70] because the temperature dependence of $J$ is neglectable. Moreover, because the Curie temperature $T_{\mathrm{c}}$ of CGT monolayer is around $60 \mathrm{~K}[66,71,72]$, here we focus on the MMI at the finite temperature below $60 \mathrm{~K}$. The magnon dispersion of CGT monolayer calculated through Eq. (16) at temperature of $0 \mathrm{~K}, 15 \mathrm{~K}, 30 \mathrm{~K}$, and $45 \mathrm{~K}$ are represented by black, red, blue and green spheres in Figure 2(a). Due to the Zeeman energy in Heisenberg Hamiltonian, an external magnetic field of 0.1T along the $c$-axis is taken into consideration in spin-wave spectrum. It can be found that the magnon frequency at finite temperature is consistent globally with the zero temperature spectra except for a slight redshift. The temperature induced redshift in magnon dispersion at finite temperature should be ascribed to the nonlinear energy $\Delta E_{\text {nonlinear }}$ caused by the nonlinear MMI. And the occurrence of nonlinear MMI is resulted by the change in magnon population at finite temperature, so that the expectation value for local $\operatorname{spin}\left\langle a_{k}^{+} a_{k}\right\rangle$ cannot be ignored.

The correction energies $\Delta E_{\text {nonlinear }}$ induced by the nonlinear MMI for optical and acoustic magnons are presented in Figure 2(b) \& 2(c). Here, we took the wave vectors $\left(k=1.03 \times 10^{6}, 1.03 \times 10^{7}, 2.06 \times 10^{7}\right.$, $3.09 \times 10^{7}, 4.12 \times 10^{7}$ and $5.15 \times 10^{7} \mathrm{~cm}^{-1}$ ) along the path from $\Gamma$ to $\mathrm{M}$ as examples. We can find that all the correction energies are negative revealing the influence of nonlinear MMI on spin-wave spectrum is to reduce the magnon frequency. The decrease in optical magnon is more pronounced than that in acoustic magnon, because the nonlinear correction energy is proportional to the magnon energy. More importantly, the nonlinear correction energies at all $k$ points become more negative with the increase of temperature, which agrees well with previous theoretical study on EuO using Dyson-Maleev theory[73]. 

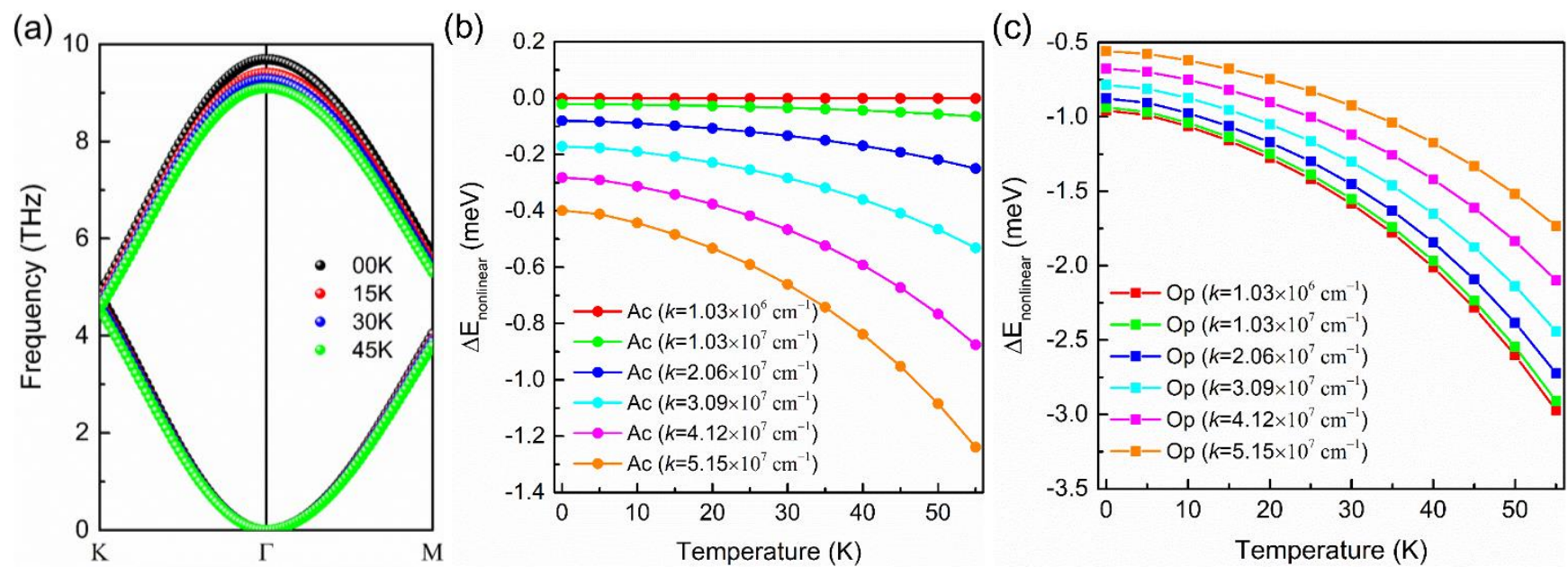

Figure 2. (a) Effects of temperature on spin wave spectrums. The nonlinear MMI correction energies for acoustic (b) and optical (c) magnons in CGT monolayer at finite temperatures. In (b \& c), wave vector $k=1.03 \times 10^{6}, 1.03 \times 10^{7}, 2.06 \times 10^{7}, 3.09 \times 10^{7}, 4.12 \times 10^{7}$ and $5.15 \times 10^{7} \mathrm{~cm}^{-1}$ are sampled along the path from $\Gamma$ to $\mathrm{M}$ in Brillouin zone.

\subsection{Magnon-magnon interaction and spin autocorrelation function}

In this work, the MMI strength is investigated by calculating the spin autocorrelation functions $<$ $S_{k}^{+}(t) S_{-k}^{-}(0)>,\left\langle S_{k}^{-}(t) S_{-k}^{+}(0)>\right.$, and $\left\langle S_{k}^{Z}(t) S_{-k}^{Z}(0)>\right.$. These spin autocorrelation functions are supposed to dominate electric spin injection, chemical potential-driven transport, which is related to the spin Seebeck effect.[70,74,75] The solutions of Heisenberg equation of motion $i \hbar \frac{\partial a_{k}\left(a_{k}^{+}\right)}{\partial t}=\hbar \omega_{k} a_{k}\left(a_{k}^{+}\right)$ based on $H(T)$ for $a_{k}(t)$ and $a_{k}^{+}(t)$ are:

$$
a_{k}(t)=a_{k}(0) e^{-i \omega_{k} t}, a_{k}^{+}(t)=a_{k}^{+}(0) e^{i \omega_{k} t}
$$

Due to the existence of external magnetic field along $c$-axis, we would put particular emphasis on the spin autocorrelation function $<S_{k}^{Z}(t) S_{-k}^{Z}(0)>$. In the reciprocal space, $S_{k}^{Z}$ can be represented as:

$$
S_{k}^{z}=N^{-\frac{1}{2}} \sum_{l} e^{-i k \cdot r_{l}} S_{l}^{z}=N^{\frac{1}{2}} S_{0} \delta_{\vec{k}, 0}-N^{-\frac{1}{2}} \sum_{k^{\prime}} a_{k^{\prime}}^{+} a_{k^{\prime}+k} .
$$


And then, $<S_{k}^{Z}(t) S_{-k}^{Z}(0)>$ can be written as:

$$
\begin{aligned}
& \left\langle S_{k}^{z}(t) S_{-k}^{z}(0)\right\rangle=\frac{\operatorname{Tr}\left\{e^{-\beta H}\left(\left(N^{\frac{1}{2}} S_{0} \delta_{k, 0}-N^{-\frac{1}{2}} \sum_{k^{\prime}} a_{k^{\prime}}^{+}(t) a_{k^{\prime}+k}(t)\right)\left(N^{\frac{1}{2}} S_{0} \delta_{-k, 0}-N^{-\frac{1}{2}} \sum_{k^{\prime}} a_{k^{+}}^{+}(0) a_{k^{\prime \prime}-k}(0)\right)\right)\right\}}{\operatorname{Tr}\left\{e^{-\beta H}\right\}} \\
& =\left\langle N S_{0}^{2} \delta_{k, 0}\right\rangle-S_{0} \delta_{k, 0}\left\langle\left(\sum_{k^{\prime}} a_{k^{\prime}}^{+}(t) a_{k^{\prime}+k}(t)+\sum_{k^{\prime}} a_{k^{-}}^{+}(0) a_{k^{\prime}-k}(0)\right)\right\rangle+N^{-1} \sum_{k^{\prime}, k^{\prime}}\left\langle a_{k^{\prime}}^{+}(t) a_{k^{\prime}+k}(t) a_{k^{\prime}}^{+}(0) a_{k^{\prime \prime}-k}(0)\right\rangle^{.} \\
& =N S_{0}^{2} \delta_{k, 0}-2 S_{0} \delta_{k, 0} \sum_{k^{\prime}}\left\langle a_{k^{\prime}}^{+}(0) a_{k^{\prime}+k}(0)\right\rangle+N^{-1} \sum_{k^{\prime}} e^{-i\left(\omega_{k^{\prime}+k^{-}} \omega_{k^{\prime}}\right) t}\left\langle a_{k^{\prime}}^{+}(0) a_{k^{\prime}+k}(0) a_{k^{\prime}+k}^{+}(0) a_{k^{\prime}}(0)\right\rangle
\end{aligned}
$$

Here, we also employed Wick's theorem to decouple these four magnon operators[63], and it is:

$$
\left\langle S_{k}^{z}(t) S_{-k}^{z}(0)\right\rangle=\left\{\begin{array}{lr}
N S_{0}^{2} \delta_{k, 0}-2 S_{0} \delta_{k, 0} \sum_{k^{\prime}}\left\langle n_{k^{\prime}}\right\rangle+N^{-1} \sum_{k^{\prime}}\left(2\left\langle n_{k^{\prime}}\right\rangle^{2}+\left\langle n_{k^{\prime}}\right\rangle\right) & (k=0) \\
N^{-1} \sum_{k^{\prime}} e^{-i\left(\omega_{k^{\prime}+k}-\omega_{k^{\prime}}\right) t}\left\langle n_{k^{\prime}}\right\rangle\left\langle n_{k^{\prime}+k}+1\right\rangle & (k \neq 0)
\end{array}\right.
$$

with $<n_{k}>=<a_{k}^{+} a_{k}>$. It is worth noting that $<n_{k}>$ is mainly contributed by acoustic magnons with respect to optical magnons, because of the low population of the latter. The spin autocorrelation function in long-wavelength limit $\left(k=5.15 \times 10^{4} \mathrm{~cm}^{-1}=0.001 \pi / \mathrm{a}\right)$ at $55 \mathrm{~K}$ and $5 \mathrm{~K}$, and under external magnetic field $B$ of $0.1 \mathrm{~T}$ are shown in Figure 3(a) \& 3(b), while that in short-wavelength limit $\left(k=5.15 \times 10^{7} \mathrm{~cm}^{-}\right.$ ${ }^{1}=1 \pi / \mathrm{a}$, with $T=55 \mathrm{~K}$ and $B=5 \mathrm{~T}$ ) is presented in Figure 3(c). As shown in Figure 3(a), it is obvious that the spin autocorrelation function decays with time and gradually converges. In order to obtain the relaxation time $\tau_{\mathrm{MM}}$ governed by magnon-magnon interaction, an exponential decay function $\left(f(t)=A \cdot \exp \left(-t / \tau_{\mathrm{MM}}\right)\right)$ was employed to fit the envelope of spin autocorrelation function, as plotted by the red solid line in Figure 3. The relaxation time $\tau_{\mathrm{MM}}$ of spin autocorrelation function can reflect the strength of MMI, and the shorter $\tau_{\mathrm{MM}}$ corresponds to a stronger MMI.

Comparing Figure 3(a) with Figure 3(b), there is a significant temperature dependence in the decay rate of spin autocorrelation function when temperature $T$ decreases from $55 \mathrm{~K}$ to $5 \mathrm{~K}$. Comparing Figure 3(a) with Figure 3(c), it can be found that the increase of wave vector $k$ from long-wavelength limit to 
short-wavelength limit causes substantial reduction in the relaxation time $\tau_{\mathrm{MM}}$. Besides, when the external magnetic field $B$ increases from $0.1 \mathrm{~T}$ to $5 \mathrm{~T}$, the decay rate of spin autocorrelation function also increases. Figure 3(d) presents the spin autocorrelation function at $k=5.15 \times 10^{4} \mathrm{~cm}^{-1}$ for $T=55 \mathrm{~K}$ and $B=0.1 \mathrm{~T}$, where only the contribution of acoustic magnons is taken into consideration. Comparing Figure 3(a) to Figure 3(d), similar results suggest the spin autocorrelation function is mainly raised from acoustic magnonmagnon interaction. Next, we systematically explore the dependence of the spin autocorrelation function and relaxation time $\tau_{\mathrm{MM}}$ on wave vector $k$, temperature $T$ and magnetic field $B$.
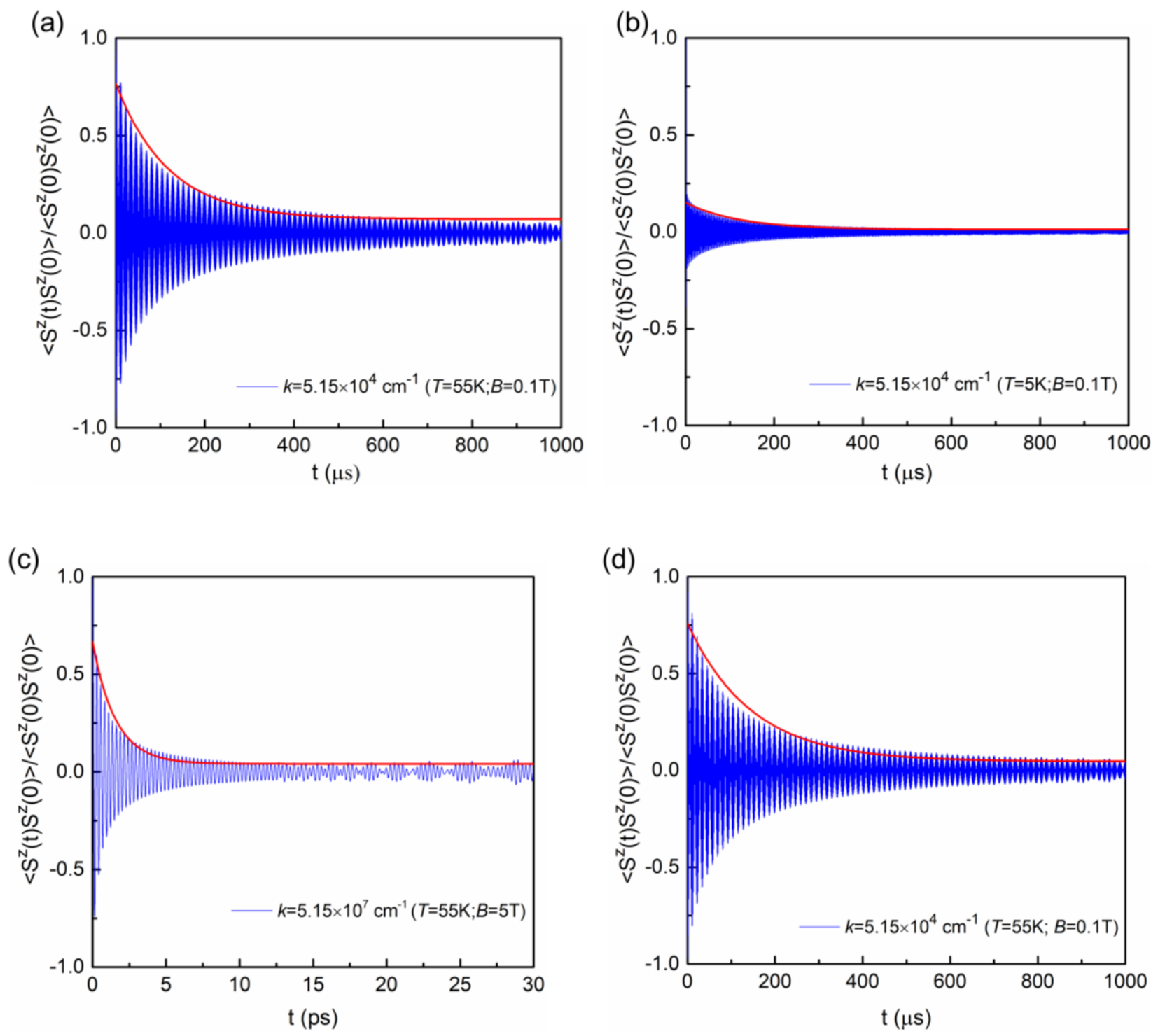
Figure 3. Autocorrelation functions at $k=5.15 \times 10^{4} \mathrm{~cm}^{-1}$ for external magnetic field $B=0.1 \mathrm{~T}$ and temperature $T=55 \mathrm{~K}$ (a) or $5 \mathrm{~K}(\mathrm{~b})$, and at $k=5.15 \times 10^{7} \mathrm{~cm}^{-1}$ for $T=55 \mathrm{~K}$ and $B=5 \mathrm{~T}$ (c), while the spin autocorrelation function only contributed by acoustic magnons at $k=5.15 \times 10^{4} \mathrm{~cm}^{-1}$ for $T=55 \mathrm{~K}$ and $B=0.1$ $\mathrm{T}$ (d). The red solid lines represent the results fitted by exponential decay function.

\subsection{Magnon relaxation time}

In Figure 4, the wave vector dependence, the temperature dependence, and the frequency dependence of the spin autocorrelation function are presented. Firstly, we focus on the wavevector-dependence. Here, we extract the relaxation time $\tau_{M M}$ based on spin autocorrelation function throughout the whole wavelength regime, temperature is fixed at $30 \mathrm{~K}$ and magnetic field is $0.1 \mathrm{~T}$, as shown in Figure 4(a). It is obvious that the relaxation time $\tau_{\mathrm{MM}}$ decreases monotonously with wavevector $k$. The energy of acoustic magnon at the boundary of Brillouin zone is much higher than that at zone center, and the higher magnon energy leads to a faster decay rate of spin autocorrelation function. It is interesting that $\tau_{\mathrm{MM}}$ at longwavelength region and short-wavelength region show different dependences on wave vector $k$, which is bounded by $k=1.03 \times 10^{6} \mathrm{~cm}^{-1}$.
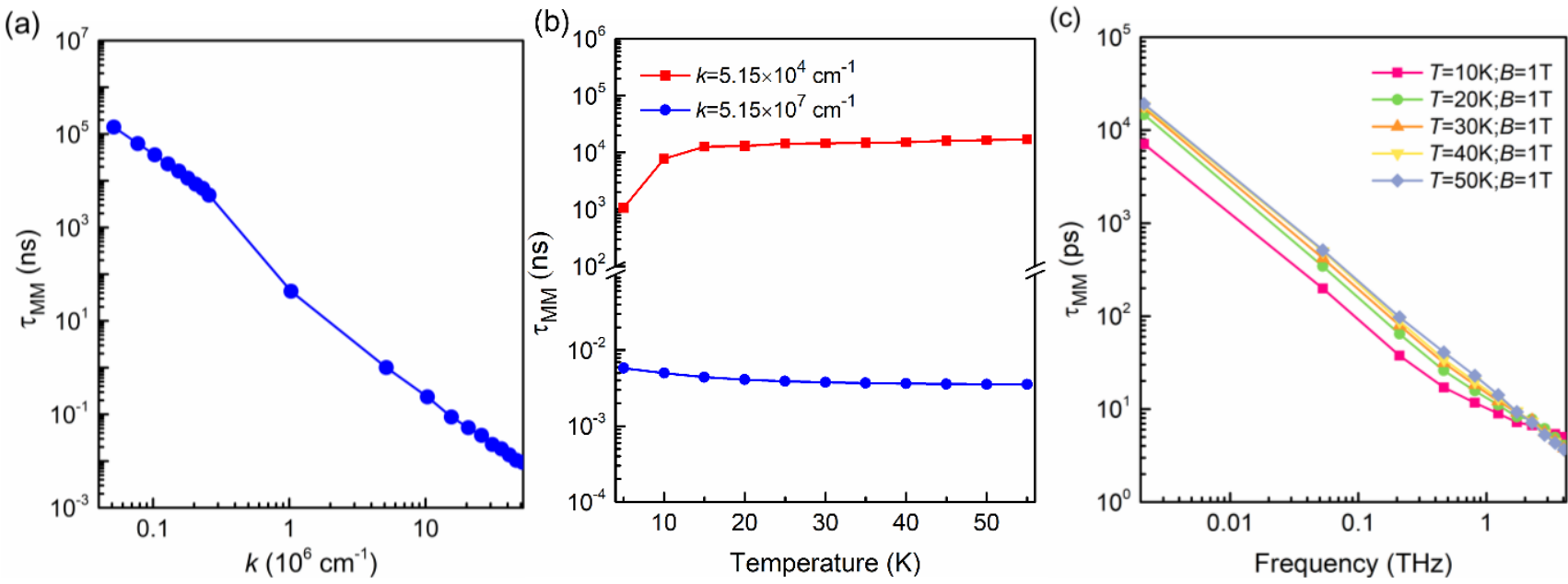
Figure 4. (a) The relaxation time $\tau_{\mathrm{MM}}$ governed by magnon-magnon interaction at $T=30 \mathrm{~K}$ and $B=0.1 \mathrm{~T}$. The curve is plotted in log-log scale. (b) The temperature dependence of $\tau_{\mathrm{MM}}$ at $k=5.15 \times 10^{4} \mathrm{~cm}^{-1}$ and $k=5.15 \times 10^{7} \mathrm{~cm}^{-1}$. Here $B=1.0 \mathrm{~T}$. (c) $\tau_{\mathrm{MM}}$ as a function of magnon frequency. Here $B=1 \mathrm{~T}$, and temperature changes from $10 \mathrm{~K}$ to $50 \mathrm{~K}$.

The temperature dependence of relaxation time $\tau_{\mathrm{MM}}$ for magnons in long-wavelength limit $(k=$ $\left.5.15 \times 10^{4} \mathrm{~cm}^{-1}=0.001 \pi / \mathrm{a}\right)$ and at the boundary of Brillouin zone $\left(k=5.15 \times 10^{7} \mathrm{~cm}^{-1}=1 \pi / \mathrm{a}\right)$ are presented in Figure 4(b). It is interesting that the relaxation times $\tau_{M M}$ in long-wavelength limit and shortwavelength limit show completely opposite temperature dependence. In long-wavelength limit, the relaxation time $\tau_{M M}$ increases with temperature, while it shows a reduction with temperature increasing at the boundary of Brillouin zone. It can be obtained from Eq. (29) that the decay rate of spin autocorrelation function depends on the magnon frequency. However, the magnon frequency decreases with temperature due to the nonlinear MMI, as shown in Figure 2. Therefore, the decay rate of spin autocorrelation function reduces with temperature, which leads to the increase in relaxation time $\tau_{\mathrm{MM}}$ for long-wavelength magnons. At the boundary of Brillouin zone, relaxation time $\tau_{M M}$ decreases with temperature due to the strong anharmonic behavior. In Figure 4(c), we present the frequency dependence of relaxation time $\tau_{\mathrm{MM}}$ under magnetic field $B=1 \mathrm{~T}$ at different temperatures $(T=10-50 \mathrm{~K})$. It is obvious that the relaxation times $\tau_{\mathrm{MM}}$ at different temperatures show similar frequency dependence. Meantime, an anharmonic behavior can be observed.

The dependences of relaxation time $\tau_{\mathrm{MM}}$ on the strength of magnetic field at $k=5.15 \times 10^{4} \mathrm{~cm}^{-1}$ and $k$ $=5.15 \times 10^{7} \mathrm{~cm}^{-1}$ at $T=30 \mathrm{~K}$ are presented in Figure $5(\mathrm{a})$. It is found that the relaxation time in both longwavelength and short-wavelength limit decreases with the enhancement of magnetic field, which is opposite with temperature dependence. Such an enhancement of the magnetic field increases the magnon 
frequency $\omega_{\boldsymbol{k}}$, resulting in the rapid decay of spin autocorrelation function. This opposite impacts between temperature and magnetic field can also be observed in Figure 6, which shows the normalized magnetic moment per $\mathrm{Cr}^{3+}$ ion at different temperature and magnetic field. The magnetic moment $M$ per $\mathrm{Cr}^{3+}$ ion is calculated by[76]:

$$
M(B, T)=g \mu_{B} \sum_{l}\left(S_{0}-\left\langle a_{l}^{+} a_{l}\right\rangle_{B, T}\right)=M_{0}\left(1-\frac{1}{N S_{0}} \sum_{k}\left\langle a_{k}^{+} a_{k}\right\rangle_{B, T}\right)
$$

where $M_{0}=g \mu_{B} S_{0}=3 \mu_{B}$ is the magnetic moment of $\mathrm{Cr}^{3+}$ at zero temperature. The dependence of normalized magnetic moment to $\mathrm{M}_{0}$ on magnetic field $B$ at different temperatures is shown in Figure 6. It is obvious that the magnetic moment grows with the enhancement of magnetic field and tends to a specific value, while it decreases close to zero with the increase of temperature. These results reveal the expectation value of local spin $<a_{k}^{+} a_{k}>$ increases with temperature but decreases with the enhancement of magnetic field[77], which is in agreement with results in Figure 4(c) \& Figure 5. Figure 5(b) presents $\tau_{\mathrm{MM}}$ as a function of frequency under different magnetic field. Overall, $\tau_{M M}$ decreases with frequency increases, independent of the strength of magnetic field.
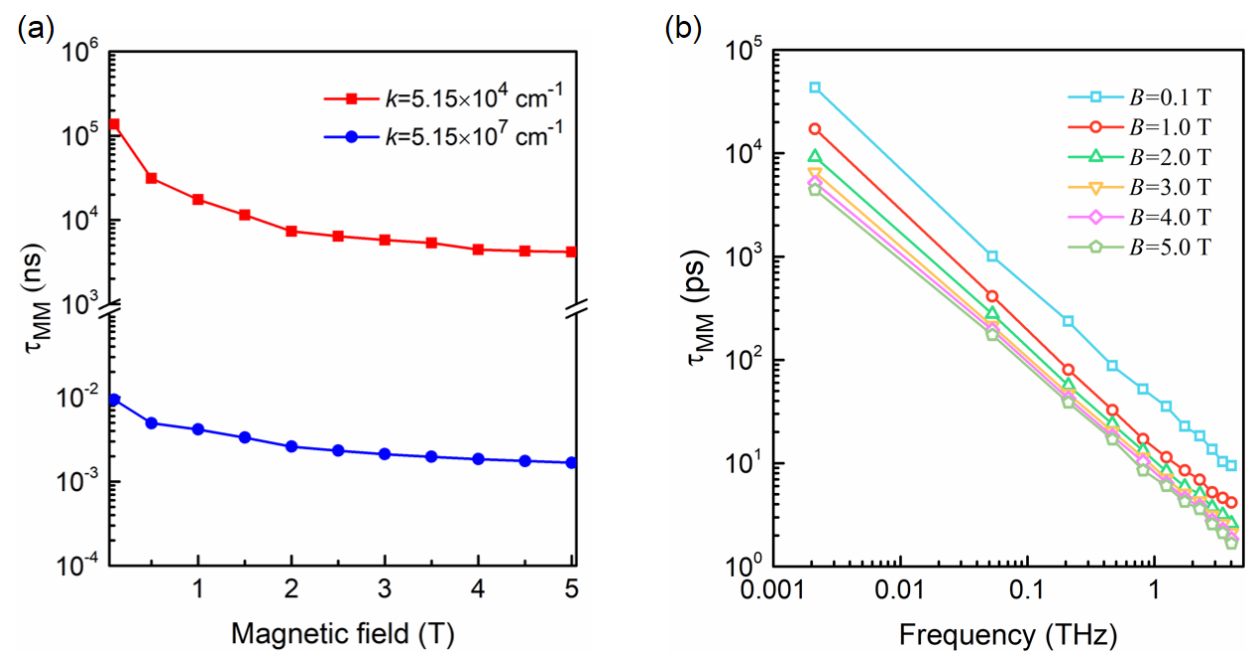
Figure 5. (a) The magnetic field dependence of $\tau_{M M}$ at $T=30 \mathrm{~K}$. (b) The frequency dependence of $\tau_{M M}$ at $T=30 \mathrm{~K}$ at different magnetic field. In (b), the $\tau_{\mathrm{MM}}$ versus frequency curves are shown in $\log -\log$ scale.

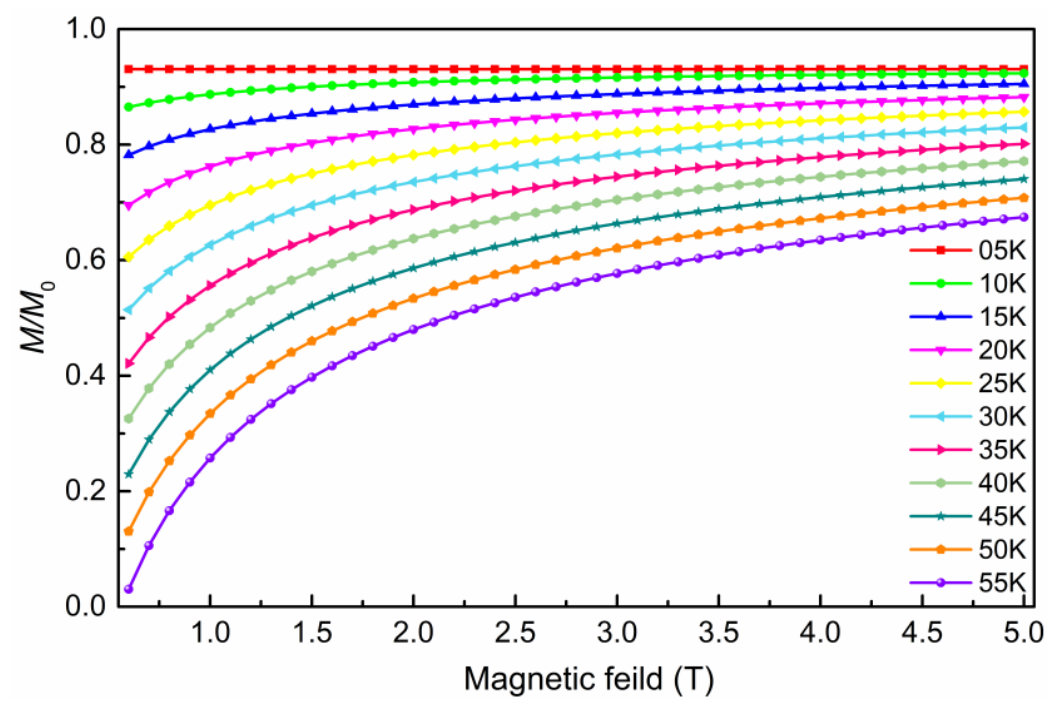

Figure 6. The magnetic moment on per $\mathrm{Cr}^{3+}$ ion normalized to $M_{0}$ versus magnetic field. Temperature changes from $5 \mathrm{~K}$ to $55 \mathrm{~K}$.

\section{Conclusions}

In summary, we established the Heisenberg Hamiltonian model for ferromagnetic 2D materials at finite temperature by taking the nonlinear magnon-magnon interaction into consideration. Based on this Hamiltonian model, it is found the increase of temperature results in a nonnegligible reduction in spinwave spectrum, especially the optical branch. Furthermore, the relaxation time $\tau_{\mathrm{MM}}$ governed by magnonmagnon interaction is also calculated based on decay of spin autocorrelation function. We find the relaxation time $\tau_{M M}$ increases with temperature but decreases with wave vector and magnetic field, because of the different magnon energy trends. All these results presented in our work are helpful for the utilization and manipulation of ferromagnetic 2D materials for applications in spintronic devices.

\section{ACKNOWLEDGEMENT}


We gratefully acknowledge the financial supports from the Graduate School of Xidian University and the China Scholarship Council (grant no. 201906960032), and the use of computing resources at the Agency for Science, Technology and Research (A*STAR) Computational Resource Centre, Singapore. 


\section{REFERENCE}

[1] A. J. Mannix, B. Kiraly, M. C. Hersam and N. P. Guisinger, Synthesis and chemistry of elemental 2D materials, Nat. Rev. Chem. 1, 1 (2017).

[2] L. Tao, E. Cinquanta, D. Chiappe, C. Grazianetti, M. Fanciulli, M. Dubey, A. Molle and D. Akinwande, Silicene field-effect transistors operating at room temperature, Nat. Nanotechnol. 10, 227 (2015).

[3] K. Novoselov, A. Mishchenko, A. Carvalho and A. C. Neto, 2D materials and van der Waals heterostructures, Science 353, aac9439 (2016).

[4] S. Manzeli, D. Ovchinnikov, D. Pasquier, O. V. Yazyev and A. Kis, 2D transition metal dichalcogenides, Nat. Rev. Mater. 2, 17033 (2017).

[5] M. Ashton, J. Paul, S. B. Sinnott and R. G. Hennig, Topology-scaling identification of layered solids and stable exfoliated 2D materials, Phys. Rev. Lett. 118, 106101 (2017).

[6] N. Mounet, M. Gibertini, P. Schwaller, D. Campi, A. Merkys, A. Marrazzo, T. Sohier, I. E. Castelli, A. Cepellotti and G. Pizzi, Two-dimensional materials from high-throughput computational exfoliation of experimentally known compounds, Nat. Nanotechnol. 13, 246 (2018).

[7] M. Gibertini, M. Koperski, A. Morpurgo and K. Novoselov, Magnetic 2D materials and heterostructures, Nat. Nanotechnol. 14, 408 (2019).

[8] C. Gong, L. Li, Z. Li, H. Ji, A. Stern, Y. Xia, T. Cao, W. Bao, C. Wang and Y. Wang, Discovery of intrinsic ferromagnetism in two-dimensional van der Waals crystals, Nature 546, 265 (2017).

[9] B. Huang, G. Clark, E. Navarro-Moratalla, D. R. Klein, R. Cheng, K. L. Seyler, D. Zhong, E. Schmidgall, M. A. McGuire and D. H. Cobden, Layer-dependent ferromagnetism in a van der Waals crystal down to the monolayer limit, Nature 546, 270 (2017).

[10] A. F. May, D. Ovchinnikov, Q. Zheng, R. Hermann, S. Calder, B. Huang, Z. Fei, Y. Liu, X. Xu and M. A. McGuire, Ferromagnetism near room temperature in the cleavable van der Waals crystal Fe5GeTe2, ACS nano 13, 4436 (2019).

[11] Z. Fei, B. Huang, P. Malinowski, W. Wang, T. Song, J. Sanchez, W. Yao, D. Xiao, X. Zhu and A. F. May, Two-dimensional itinerant ferromagnetism in atomically thin Fe3GeTe2, Nat. Mater. 17, 778 (2018).

[12] Y. Shiomi, R. Takashima and E. Saitoh, Experimental evidence consistent with a magnon Nernst effect in the antiferromagnetic insulator MnPS3, Phys. Rev. B 96, 134425 (2017). 
[13] G. Long, H. Henck, M. Gibertini, D. Dumcenco, Z. Wang, T. Taniguchi, K. Watanabe, E. Giannini and A. F. Morpurgo, Persistence of magnetism in atomically thin MnPS3 crystals, Nano Lett. 20, 2452 (2020).

[14] S. Kim, J. Lee, G. Jin, M.-H. Jo, C. Lee and S. Ryu, Crossover between Photochemical and Photothermal Oxidations of Atomically Thin Magnetic Semiconductor CrPS4, Nano Lett. 19, 4043 (2019). [15] A. K. Budniak, N. A. Killilea, S. J. Zelewski, M. Sytnyk, Y. Kauffmann, Y. Amouyal, R. Kudrawiec, W. Heiss and E. Lifshitz, Exfoliated CrPS4 with Promising Photoconductivity, Small 16, 1905924 (2020).

[16] A. McCreary, J. R. Simpson, T. T. Mai, R. D. McMichael, J. E. Douglas, N. Butch, C. Dennis, R. V. Aguilar and A. R. H. Walker, Quasi-two-dimensional magnon identification in antiferromagnetic FePS3 via magneto-Raman spectroscopy, Phys. Rev. B 101, 064416 (2020).

[17] W. Yang, A. L. Coughlin, L. Webster, G. Ye, K. Lopez, H. A. Fertig, R. He, J.-A. Yan and S. Zhang, Highly tunable Raman scattering and transport in layered magnetic Cr2S3 nanoplates grown by sulfurization, 2D Mater. 6, 035029 (2019).

[18] F. Cui, X. Zhao, J. Xu, B. Tang, Q. Shang, J. Shi, Y. Huan, J. Liao, Q. Chen and Y. Hou, Controlled Growth and Thickness-Dependent Conduction-Type Transition of 2D Ferrimagnetic Cr2S3 Semiconductors, Adv. Mater. 32, 1905896 (2020).

[19] S. Zhang, R. Xu, W. Duan and X. Zou, Intrinsic Half-Metallicity in 2D Ternary Chalcogenides with High Critical Temperature and Controllable Magnetization Direction, Adv. Funct. Mater. 29, 1808380 (2019).

[20] Q. Wu, Y. Zhang, Q. Zhou, J. Wang and X. C. Zeng, Transition-Metal Dihydride Monolayers: A New Family of Two-Dimensional Ferromagnetic Materials with Intrinsic Room-Temperature HalfMetallicity, J. Phys. Chem. Lett. 9, 4260 (2018).

[21] T. Zhang, Y. Wang, H. Li, F. Zhong, J. Shi, M. Wu, Z. Sun, W. Shen, B. Wei and W. Hu, Magnetism and Optical Anisotropy in van der Waals Antiferromagnetic Insulator CrOCl, ACS nano 13, 11353 (2019).

[22] N. Miao, B. Xu, L. Zhu, J. Zhou and Z. Sun, 2D intrinsic ferromagnets from van der Waals antiferromagnets, J. Am. Chem. Soc. 140, 2417 (2018).

[23] A. Bandyopadhyay, N. C. Frey, D. Jariwala and V. B. Shenoy, Engineering Magnetic Phases in Two-Dimensional Non-van der Waals Transition-Metal Oxides, Nano Lett. 19, 7793 (2019). 
[24] J. He, G. Ding, C. Zhong, S. Li, D. Li and G. Zhang, Cr2TiC2-based double MXenes: novel 2D bipolar antiferromagnetic semiconductor with gate-controllable spin orientation toward antiferromagnetic spintronics, Nanoscale 11, 356 (2019).

[25] N. C. Frey, A. Bandyopadhyay, H. Kumar, B. Anasori, Y. Gogotsi and V. B. Shenoy, SurfaceEngineered MXenes: Electric Field Control of Magnetism and Enhanced Magnetic Anisotropy, ACS nano 13, 2831 (2019).

[26] N. D. Mermin and H. Wagner, Absence of ferromagnetism or antiferromagnetism in one-or twodimensional isotropic Heisenberg models, Phys. Rev. Lett. 17, 1133 (1966).

[27] Y. Li, Z. Jiang, J. Li, S. Xu and W. Duan, Magnetic anisotropy of the two-dimensional ferromagnetic insulator MnBi2Te4, Phys. Rev. B 100, 134438 (2019).

[28] I. Lee, F. G. Utermohlen, D. Weber, K. Hwang, C. Zhang, J. van Tol, J. E. Goldberger, N. Trivedi and P. C. Hammel, Fundamental Spin Interactions Underlying the Magnetic Anisotropy in the Kitaev Ferromagnet CrI3, Phys. Rev. Lett. 124, 017201 (2020).

[29] L. Webster and J.-A. Yan, Strain-tunable magnetic anisotropy in monolayer $\mathrm{CrCl} 3, \mathrm{CrBr} 3$, and CrI3, Phys. Rev. B 98, 144411 (2018).

[30] J. A. Sears, L. E. Chern, S. Kim, P. J. Bereciartua, S. Francoual, Y. B. Kim and Y.-J. Kim, Ferromagnetic Kitaev interaction and the origin of large magnetic anisotropy in $\alpha$-RuCl3, Nat. Phys., 1 (2020).

[31] C. Xu, J. Feng, H. Xiang and L. Bellaiche, Interplay between Kitaev interaction and single ion anisotropy in ferromagnetic CrI3 and CrGeTe3 monolayers, npj Computational Materials 4, 1 (2018).

[32] H. Kumar, N. C. Frey, L. Dong, B. Anasori, Y. Gogotsi and V. B. Shenoy, Tunable magnetism and transport properties in nitride MXenes, ACS nano 11, 7648 (2017).

[33] N. C. Frey, H. Kumar, B. Anasori, Y. Gogotsi and V. B. Shenoy, Tuning noncollinear spin structure and anisotropy in ferromagnetic nitride MXenes, ACS nano 12, 6319 (2018).

[34] M. Rassekh, J. He, S. F. Shayesteh and J. J. Palacios, Remarkably enhanced Curie temperature in monolayer CrI3 by hydrogen and oxygen adsorption: A first-principles calculations, Comp. Mater. Sci. 183, 109820 (2020).

[35] Z. Wang, T. Zhang, M. Ding, B. Dong, Y. Li, M. Chen, X. Li, J. Huang, H. Wang and X. Zhao, Electric-field control of magnetism in a few-layered van der Waals ferromagnetic semiconductor, Nat. Nanotechnol. 13, 554 (2018). 
[36] K. Wang, T. Hu, F. Jia, G. Zhao, Y. Liu, I. V. Solovyev, A. P. Pyatakov, A. K. Zvezdin and W. Ren, Magnetic and electronic properties of $\mathrm{Cr} 2 \mathrm{Ge} 2 \mathrm{Te} 6$ monolayer by strain and electric-field engineering, Appl. Phys. Lett. 114, 092405 (2019).

[37] X.-J. Dong, J.-Y. You, B. Gu and G. Su, Strain-induced room-temperature ferromagnetic semiconductors with large anomalous Hall conductivity in two-dimensional Cr2Ge2Se6, Physical Review Applied 12, 014020 (2019).

[38] Y. Fang, S. Wu, Z.-Z. Zhu and G.-Y. Guo, Large magneto-optical effects and magnetic anisotropy energy in two-dimensional Cr2Ge2Te6, Phys. Rev. B 98, 125416 (2018).

[39] M. Wu, Z. Li, T. Cao and S. G. Louie, Physical origin of giant excitonic and magneto-optical responses in two-dimensional ferromagnetic insulators, Nat. Commun. 10, 1 (2019).

[40] K. Shen, Magnon Spin Relaxation and Spin Hall Effect Due to the Dipolar Interaction in Antiferromagnetic Insulators, Phys. Rev. Lett. 124, 077201 (2020).

[41] J. Li, Y. Li, S. Du, Z. Wang, B.-L. Gu, S.-C. Zhang, K. He, W. Duan and Y. Xu, Intrinsic magnetic topological insulators in van der Waals layered MnBi2Te4-family materials, Sci. Adv. 5, eaaw5685 (2019). [42] M. Lohmann, T. Su, B. Niu, Y. Hou, M. Alghamdi, M. Aldosary, W. Xing, J. Zhong, S. Jia and W. Han, Probing magnetism in insulating Cr2Ge2Te6 by induced anomalous Hall effect in Pt, Nano Lett. 19, 2397 (2019).

[43] M.-G. Han, J. A. Garlow, Y. Liu, H. Zhang, J. Li, D. DiMarzio, M. W. Knight, C. Petrovic, D. Jariwala and Y. Zhu, Topological magnetic-spin textures in two-dimensional van der Waals Cr2Ge2Te6, Nano Lett. 19, 7859 (2019).

[44] R. Samajdar, M. S. Scheurer, S. Chatterjee, H. Guo, C. Xu and S. Sachdev, Enhanced thermal Hall effect in the square-lattice Néel state, Nat. Phys. 15, 1290 (2019).

[45] S. Park, N. Nagaosa and B.-J. Yang, Thermal Hall Effect, Spin Nernst Effect, and Spin Density Induced by a Thermal Gradient in Collinear Ferrimagnets from Magnon-Phonon Interaction, Nano Lett. 20, 2741 (2020).

[46] L. Du, J. Tang, Y. Zhao, X. Li, R. Yang, X. Hu, X. Bai, X. Wang, K. Watanabe and T. Taniguchi, Lattice Dynamics, Phonon Chirality, and Spin-Phonon Coupling in 2D Itinerant Ferromagnet Fe3GeTe2, Adv. Funct. Mater. 29, 1904734 (2019).

[47] J. E. Rodrigues, W. S. Rosa, M. M. Ferrer, T. R. Cunha, M. J. M. Zapata, J. R. Sambrano, J. L. Martínez, P. S. Pizani, J. A. Alonso and A. C. Hernandes, Spin-phonon coupling in uniaxial anisotropic spin-glass based on Fe2TiO5 pseudobrookite, J. Alloys Compd. 799, 563 (2019). 
[48] L. Webster, L. Liang and J.-A. Yan, Distinct spin-lattice and spin-phonon interactions in monolayer magnetic CrI3, Phys. Chem. Chem. Phys. 20, 23546 (2018).

[49] B. Zhang, Y. Hou, Z. Wang and R. Wu, First-principles studies of spin-phonon coupling in monolayer Cr2Ge2Te6, Phys. Rev. B 100, 224427 (2019).

[50] L.-W. Wang, L.-S. Xie, P.-X. Xu and K. Xia, First-principles study of magnon-phonon interactions in gadolinium iron garnet, Phys. Rev. B 101, 165137 (2020).

[51] K. Wang, J. He, M. Zhang, H. Wang and G. Zhang, Magnon-phonon interaction in antiferromagnetic two-dimensional MXenes, Nanotechnology 31, 435705 (2020).

[52] P. Jiang, C. Wang, D. Chen, Z. Zhong, Z. Yuan, Z.-Y. Lu and W. Ji, Stacking tunable interlayer magnetism in bilayer CrI3, Phys. Rev. B 99, 144401 (2019).

[53] K. Guo, B. Deng, Z. Liu, C. Gao, Z. Shi, L. Bi, L. Zhang, H. Lu, P. Zhou and L. Zhang, Layer dependence of stacking order in nonencapsulated few-layer CrI3, Science China Materials 63, 413 (2020).

[54] S. Jiang, L. Li, Z. Wang, K. F. Mak and J. Shan, Controlling magnetism in 2D CrI3 by electrostatic doping, Nat. Nanotechnol. 13, 549 (2018).

[55] H. Tsai, T. Higo, K. Kondou, T. Nomoto, A. Sakai, A. Kobayashi, T. Nakano, K. Yakushiji, R. Arita and S. Miwa, Electrical manipulation of a topological antiferromagnetic state, Nature 580, 608 (2020).

[56] T. Song, Z. Fei, M. Yankowitz, Z. Lin, Q. Jiang, K. Hwangbo, Q. Zhang, B. Sun, T. Taniguchi and K. Watanabe, Switching 2D magnetic states via pressure tuning of layer stacking, Nat. Mater., 1 (2019).

[57] T. Li, S. Jiang, N. Sivadas, Z. Wang, Y. Xu, D. Weber, J. E. Goldberger, K. Watanabe, T. Taniguchi and C. J. Fennie, Pressure-controlled interlayer magnetism in atomically thin CrI3, Nat. Mater. 18, 1303 (2019).

[58] L. R. Mead and N. Papanicolaou, Holstein-Primakoff theory for many-body systems, Phys. Rev. B 28, 1633 (1983).

[59] M. M. Nieto and D. R. Truax, Holstein-Primakoff/Bogoliubov Transformations and the Multiboson System, Fortschr. Phys. 45, 145 (1997).

[60] S. Halilov, A. Perlov, P. Oppeneer and H. Eschrig, Magnon spectrum and related finitetemperature magnetic properties: A first-principle approach, EPL (Europhysics Letters) 39, 91 (1997).

[61] S. Halilov, H. Eschrig, A. Y. Perlov and P. Oppeneer, Adiabatic spin dynamics from spin-densityfunctional theory: Application to Fe, Co, and Ni, Phys. Rev. B 58, 293 (1998). 
[62] D. Pushkarov and K. I. Pushkarov, Solitary magnons in one-dimensional ferromagnetic chain, Phys. Lett. A 61, 339 (1977).

[63] G.-C. Wick, The evaluation of the collision matrix, Phys. Rev. 80, 268 (1950).

[64] V. Carteaux, D. Brunet, G. Ouvrard and G. Andre, Crystallographic, magnetic and electronic structures of a new layered ferromagnetic compound Cr2Ge2Te6, J. Phys.: Condens. Matter 7, 69 (1995).

[65] G. Kresse, J. Furthmüller, Efficient iterative schemes for ab initio total-energy calculations using a plane-wave basis set. Phy. Rev. B 54 (16), 11169-11186 (1996).

[66] J. He, G. Ding, C. Zhong, S. Li, D. Li and G. Zhang, Remarkably enhanced ferromagnetism in a super-exchange governed Cr2Ge2Te6 monolayer via molecular adsorption, J. Mater. Chem. C 7, 5084 (2019).

[67] G. Menichetti, M. Calandra and M. Polini, Electronic structure and magnetic properties of fewlayer Cr2Ge2Te6: the key role of nonlocal electron-electron interaction effects, 2D Mater. 6, 045042 (2019).

[68] J. Kanamori, Superexchange interaction and symmetry properties of electron orbitals, J. Phys. Chem. Solids 10, 87 (1959).

[69] K. Shen, Finite temperature magnon spectra in yttrium iron garnet from a mean field approach in a tight-binding model, New J. Phys. 20, 043025 (2018).

[70] J. Barker and G. E. Bauer, Thermal spin dynamics of yttrium iron garnet, Phys. Rev. Lett. 117, 217201 (2016).

[71] Y. Liu and C. Petrovic, Critical behavior of quasi-two-dimensional semiconducting ferromagnet Cr2Ge2Te6, Phys. Rev. B 96, 054406 (2017).

[72] N. Ito, T. Kikkawa, J. Barker, D. Hirobe, Y. Shiomi and E. Saitoh, Spin Seebeck effect in the layered ferromagnetic insulators CrSiTe3 and CrGeTe3, Phys. Rev. B 100, 060402 (2019).

[73] O. Dietrich, J. Als-Nielsen and L. Passell, Neutron scattering from the Heisenberg ferromagnets EuO and EuS. III. Spin dynamics of EuO, Phys. Rev. B 14, 4923 (1976).

[74] L. Cornelissen, J. Liu, R. Duine, J. B. Youssef and B. Van Wees, Long-distance transport of magnon spin information in a magnetic insulator at room temperature, Nat. Phys. 11, 1022 (2015).

[75] L. J. Cornelissen, K. J. Peters, G. E. Bauer, R. Duine and B. J. van Wees, Magnon spin transport driven by the magnon chemical potential in a magnetic insulator, Phys. Rev. B 94, 014412 (2016).

[76] F. Bloch, Zur theorie des ferromagnetismus, Z. Phys. 61, 206 (1930). 
[77] D. R. Klein, D. MacNeill, J. L. Lado, D. Soriano, E. Navarro-Moratalla, K. Watanabe, T. Taniguchi, S. Manni, P. Canfield and J. Fernández-Rossier, Probing magnetism in 2D van der Waals crystalline insulators via electron tunneling, Science 360, 1218 (2018). 